THE WABASH CENTER

JOURNAL on TEACHING

\title{
A Guide to Collaborative Communication for Service- Learning and Community Engagement Partners
}

\author{
Rebecca J. Dumlao \\ Sterling, VA: Stylus Publishing, 2018 (192 pages, ISBN 978-1620361085, \$27.50)
}

\section{Reviewed By}

AHyun Lee

Wesley Seminary at Indiana Wesleyan University
Rebecca J. Dumlao engages in collaborative communication and offers strategies and knowledge through real-life examples to build service-learning and community engagement partnerships.

Chapter 1 focuses on "being and doing collaboratively" in individual interactions. Dumlao focuses on who a person is and how self-reflection impacts their communication with others. Communication is the integral process of sharing and understanding meaning while maintaining collaborative efforts (Pearson and Nelson, An Introduction to Human Communication [McGraw-Hill, 2000], 6).

Dumlao describes these experiences as the foundation of partnership communication in chapter 2 . It begins with a partnership between two people who share common goals of trust and respect. Collaboration examines how people think, interact, and work with each other to build a brighter future.

Chapter 3 lists the five frameworks of collaborative communication: (1) Connect and build trusting, effective relationships; (2) Converse in ways that participants are heard and understood; (3) Envision the possibilities of what can be done together; (4) Commit to one another and the joint work toward creating solutions; and (5) Understand partner patterns that make this relationship unique. Chapter 4 includes specific practices and collaborative communication processes by applying these five frameworks.

In chapter 5 , collaboration requires communication practices which extend the partnership to the community. Dumlao integrates the framework of collaborative communication with topics of cultural differences and conflict management while supporting collaborative relationships in chapters 6 and 7 . She provides practical strategies for creating a safe space where people tell the truth, listen empathically to other's experiences, and form a supportive communication process.

Chapter 8 introduces creative ways to celebrate the partnership, affirm accomplishment with collaborative communication, and extend community engagement through storytelling, artwork, webinars, and short audios and videos.

How does using the framework of collaborative communication construct and impact the intersectionality of multiple and ever-shifting identities? Partnerships need to be understood beyond individuals because communication also conveys institutional and social expectations. Focusing on interpersonal partnership often overlooks the reciprocal and mutual accountability of individual, interpersonal, and communal communication.

In sum, collaborative communication can happen when individuals listen to each other with "relational empathy." This involves mutual creativity in communication and leads to a "third culture" (109) where relational empathy forms unique values and norms through conversations, and service-learning and community engagement transforms culture. This book is valuable for empowering partnerships at all levels for those who want to work more effectively in relationships and community through building service-learning and community engagement.

296 2021; 2:1 296-296 The Wabash Center Journal on Teaching

This work is licensed under a Creative Commons Attribution-NonCommercial 4.0 International License 